4. Es empfiehlt sich zwar, überall auf eine richtige und sachgemäße Einrichtung der einzelnen Wasserversorgungsquellen zu achten und mangelhafte Einrichtungen dieser Art zu beseitigen; indes ist es durch eine blobe Ortsbesichtigung nur möglich, dieses festzustellen und weiter, ob offensichtliche Verunreinigungen stattfinden; die unterirdisch vor sich gehenden Verunreinigungen geben sich durch eine Augenscheinnahme allein nicht kund; auch genügen zu deren Feststellung nicht einige qualitative Reaktionen, auch nicht die Ermittelung der Auzahl der Bakterienkeime; über diese Art Verunreinigung kann nur eine genaue quantitative chemische Analyse des Wassers Aufschluß geben. Alle Äußerungen und Verordnungen (z. B. die in der Dienstanweisung für die preußischen Kreisärzte vom 23. März 1901 No. 3), wonach der Schwerpunkt der Beurteilung eines Wassers weniger auf die chemische und bakteriologische Untersuchung von Wasserproben als auf die örtliche Besichtigung gelegt werden soll, sind aus wissenschaftlichen wie praktischen Gründen unhaltbar und verwerflich.

5. Die örtliche Besichtigung einer Wasserversorgungsquelle ist durchweg erwünscht und in allen besonders wichtigen Fällen notwendig; der Arzt besitzt dazu indes gewilo keine bessere Befähigung als der Chemiker oder Hydrotechniker, ausgenommen jene Fälle, in welchen eine Infektion mit den Erregern menschlicher Infektionskrankheiten vorliegt oder vorliegen soll; diese Fälle kommen indes seltener in Betracht, als gegenwärtig angenommen wird. Jedenfalls soll die Ortsbesichtigung und Probenahme von dem ausgeführt werden, der auch die maßgebende Untersuchung auszufubren hat.

6. Die chemische Analyse eines Wassers hat nur dann einen wirklichen und vollen Wert, wenn sie sich auf alle jeweilig in Frage kommenden Bestandteile des Wassers erstreckt und die Ergebnisse eine sinn- und sachgemäße Erklärung finden."

Daran schloß sich das Korreferat:

\title{
Über die Beurteilung des Wassers vom bakteriologischen Standpunkte.
}

\author{
Von
}

\section{Prof. Dr. R. Emmerich ${ }^{1}$ ) in München.}

Im Jahre 1880 hat sich die Stadt München für die Versorgung der Stadt mit Wasser aus dem Mangfalltale entschieden und eine großartige Wasserleitung hergestellt, die in bezug auf Qualität und Quantität des Wassers als eine geradezu ideale bezeichnet werden muß, um welche München von der ganzen Welt beneidet wird.

Die Wahl dieses Wassers ist dem Zusammenwirken der Hydrotechniker Salbach, Thiem, des Geologen v. Gümbel und des Chemikers und Hygienikers von Pettenk ofer zu verdanken.

Die bakteriologischen Untersuchungsmethoden des Wassers waren damals noch nicht bekannt, da die Wissenschaft erst zwei Jahre später durch Robert Koch damit beschenkt wurde.

1) Die in diesem Referat erwähnten Untersuchungen über die Vernichtung von pathogenen Bakterien im Wasser durch Flagellaten wurden gemeinschaftlich mit Privatdozent Dr. Ge $\mathrm{m}$ ü $\mathrm{n} d$ ansgeführt. 
Die Wasserversorgung Münchens ist ein Beweis dafür, daß man auch ohne Zuhilfenahme der bakteriologischen Untersuchung im stande ist, unter den einer Gegend zur Verfügung stehenden Wasserbezugsquellen die reinste und geeignetste auszuwählen; denn das Münchener Mangfallwasser ist nicht nur in physikalischer und chemischer, sondern auch in bakteriologischer Beziebung als vollkommen rein zu bezeichnen, da es nur 2-10 (in seltenen Fällen 20) Keime in $1 \mathrm{cem}$ enthält, die zwei, höchstens drei Arten angehören. Außerdem finden sich im Mangfallwasser noch 2 Arten von Flagellaten (Bodo saltans und Bodo ovatus), die, wie ich später zeigen werde, die sanitätspolizeiliche Schutzwehr des Wassers darstellen. Das ist alles an Lebewesen in diesem reinen und wohlschmeckenden Wasser und doch verdanken wir seine Wahl nicht der bakteriologischen, sondern nur der hydrotechnischen, geologischen und chemischen Untersuchung.

Aber, meine Herren, nicht jeder Chemiker oder Hygieniker ist ein Pettenkof er. Und wie dieser und seine älteren Fachgenossen alle Untersuchungsmethoden, die über die Beschaffenheit eines Wassers Aufschluß geben können, stets herangezogen haben, so werden auch wir in wichtigen Fällen von allen bisher benutzten Untersuchungsverfahren Gebrauch machen und die bakteriologischen Untersuchungsmethoden als eine auBerordentlich wertvolle Unterstützung für die Beurteilung des Wassers in den meisten Fällen verwerten. Die Frage, welche Untersuchungsverfahren für die Beurteilung des Wassers den meisten Wert haben: die physikalischen, chemischen, bakteriologischen, oder die Augenscheinnahme - diese Frage ist eigentlich eine müßige Frage; denn es kommt ganz auf den einzelnen Fall und auf die Fragestellung an, welcher von diesen Untersuchungsarten wir die wesentlichste Bedeutung zuerkennen müssen.

Herr Prof. König hat heute in sehr zutreffender Weise gezeigt, daß für die Lösung von Fragen, welche die gewerbliche und industrielle Verwendung des Wassers betreffen, die chemische Untersuchung meistens den Hauptwert hat und dal sie in manchen von diesen Fällen allein entscheidet.

Es gibt aber andererseits auch Fragen, für deren Beantwortung die bakteriologische Untersuchung von größter Wichtigkeit ist, allerdings ohne daß hierbei die physikalische, chemische und mikroskopische Untersuchung überflüssig werden. Es ist dies bekanntlich dann der Fall, wenn eine Wasserbezugsquelle beschuldigt wird, eine Infektionskrankheit oder eine Epidemie verursacht zu haben. Diese Kardinalfrage der Bakteriologie und Hygiene, bei deren Würdigung sich die Ansichten der Kontagionisten und Trinkwassertheoretiker einerseits und der Lokalisten andererseits d. h. der Koch'schen und der Pettenk ofer'schen Schule schroff gegenüberstehen, soll heute noch eingehender besprochen werden. Vorher aber muß ich ein Hauptprinzip in dem Satze formulieren:

Zur Beurteilung eines Wassers, das für die Versorgung einer Stadt oder Ortschaft oder eines Komplexes von solehen bestimmt ist, sind die notwendigen hydrotechnischen, geologischen und physikalischen Untersuchungen, die Augenscheinnahme inbegriffen, sowiediechemische, bakteriologische und mikroskopische Analyse auszuführen und so oft zu wiederholen als dies zur Feststellung der möglichen Schwankungen in bezug auf Qualität und Quantität nötig ist.

Da ich mich heute nur mit der bakteriologischen und mikroskopischen Unter- 
suchung zu befassen habe, so beschränke ich mich darauf hervorzuheben, daß in diesen für die Gesundheit großer Menschenmassen so wichtigen Fällen, die bakteriologische und mikroskopische Untersuchung von gleicher Bedeutung wie die chemische Analyse sind.

Noch mehr als bei der chemischen Analyse ist bei der Ausführung der bakteriologischen Untersuchung eine große Übung und Erfahrung unbedingte Voraussetzung. Leider sind die in den häuslichen Abwässern, den Aborten und Dunggruben etc. vorkommenden Bakterienarten noch nicht genügend studiert. Während sich eine Verunreinigung des Wassers mit menschlichen oder tierischen Exkrementen aus dem Kochsalz-, Salpetersäuregehalt u. s. w. des Wassers beim Vergleich mit der chemischen Beschaffenheit des Normalwassers der Gegend mit Sicherheit ersehen läßt, gibt die bakteriologische Untersuchung hierfür weniger sichere Anhaltspunkte; denn selbst ein reichliches Vorkommen von Bacterium coli commune im Wasser darf nur mit groBer Vorsicht zu dem Schlusse verwertet werden, daß menschliche oder tierische Exkremente ohne genügende Filtration durch die Rinnsale des Bodens in das Wasser gelangen. Man wird diesen Schluf immer nur dann ziehen, wenn er auch durch die Augenscheinnahme und die chemische Untersuchung unterstützt wird. In dieser Beziehung wird von wenig erfahrenen Bakteriologen viel Mißbrauch getrieben, der den Städten oft viele Kosten und Mißlichkeiten verursacht. Ich kenne einen Fall, bei welchem sich ein Bakteriologe gegen die Verwendung eines ganz reinen, auf Grund der chemischen Untersuchung als völlig einwandfrei zu bezeichnenden Wassers mit Entschiedenheit ausgesprochen hat, vermeintlich weil 4 oder 5 Kolonien von Bacterium coli commune aus $1 \mathrm{ccm}$ Wasser zur Entwickelung kamen.

Die betreffende Stadt führte darauf hin neue Bohrungen aus, aber auch in dem neugebohrten Wasser fand sich das vermeintliche Bacterium coli. Dies wurde dem mit dem Projekt betrauten Hydrotechniker doch zu bunt, zumal über dem Grundwasser eine 4 bis 5 Meter mächtige Schicht von reinem Sand und feinem Kies gelagert war. Ich wurde nun ebenfalls mit der Untersuchung des Wassers beauftragt und konnte feststellen, daß auf den damit besäten Gelatineplatten allerdings koliähnliche Kolonien zur Entwickelung kamen, die aber nach Übertragung in Bouillon bei $37^{\circ}$ kein Wachstum zeigten. Es handelte sich also, wie auch noch durch weitere Untersuchungen festgestellt wurde, überhaupt nicht um Bacterium coli, sondern um eine der zahlreichen verwandten Arten, welche ähnliche Kolonien auf Gelatine bilden und in der Luft, im Boden etc. sehr häufig vorkommen.

Mit dem angeblichen Nachweis von Bacterium coli im Wasser wird also sehr oft Mißbrauch getrieben und Schaden anstatt Nutzen gestiftet.

Der Befund von Bacterium coli im Brunnenwasser wird sehr oft verzeichnet und daraus geschlossen, daß das Wasser mit menschlichen oder tierischen Exkrementen verunreinigt sei.

$\mathrm{Ob}$ es sich dabei immer um diese Bakterienart gehandelt hat, ist höchst zweifelhaft ${ }^{1}$ ). Die Identifizierung des Bacterium coli ist gar nicht so leicht, sie macht

1) In Wasser, Boden, Luft ete. kommen sehr viele Bakterienarten vor, die dem Bacterium coli sehr ähnlich, aber doch nicht damit identisch sind. Es ist therhaupt unwahrscheinlich, daf3 Bacterium coli auf unterirdischem Wege bäufig ins Brunnenwasser gelangt; denn dieses würde voraussetzen, dafb freie Rinnsale zwischen Abortgruben und den Brunnen vorbanden sind oder daßs der Boden im Bereich des Grundwassers grofe Mengen dieser Bakterienart enthält, was bekanntlich selten der Fall ist. Leichter kann diese Bakterienart bei mangelhafter Brunnenbedeckung den Weg ins Wasser finden. 
ebensoviel Untersuchungen nötig, wie die Identifizierung des Typhusbacillus und man sollte den Nachweis nie als erbracht ansehen, ehe nicht auch die Agglutinationsprobe positiv ausgefallen ist.

Bis jetzt kennen wir nur wenige Bakterienarten, deren Nachweis nach dem gegenwärtigen Stande unseres Wissens eine symptomatische Bedeutung zugesprochen werden könnte. Die Zahl dieser Arten wird sich aber, wenn einmal die Bakterienflora der verschiedenen $A b w a ̈ s s e r$ und Abfälle genauer untersucht ist, erhöhen und die bakteriologische Untersuchung des Wassers wird hierdurch an Wert gewinnen.

Große Erwartungen wurden an die bakteriologische Wasseruntersuchung bezüglich der Klarlegung der Ätiologie gewisser Infektionskrankheiten, insbesondere beim $\mathrm{Ab}$ dominaltyphus, der Cholera und Malaria geknüpft. Von der Malaria wissen wir jetzt bestimmt, daß sie durch das Trinkwasser nicht verbreitet wird. In üherraschender Weise wurde die Überimpfung der Malariaparasiten durch die Anopheles-Arten klargelegt.

Man braucht kein Prophet zu sein, um sagen zu können, då in ähnlicher Weise wie bei der Malaria in nicht zu ferner Zeit auch für Typhus und Cholera der bakteriologische Beweis erbracht werden wird, daß ihre Entstehung und Verbreitung mit dem Wasser nichts zu schaffen hat.

Pettenkofer hat bekanntlich mit aller Entschiedenheit diesen Standpunkt schon lange vertreten und er hat gezeigt, dah viele schwere Typhus- und Choleraepidemien, namentlich auch die in München, unmöglich durch Trinkwasser verursacht sein konnten. Die Argumente Pettenk ofer's sind so zahlreich, so schlagend und bis jetzt noch so unersehüttert, daß für den Kenner dieser Arbeiten der epidemiologisehe Beweis von der Bedeutungslosigkeit des Wassers für die Entstehung von Typhus und Cholera bereits erbracht ist.

Merkwürdigerweise haben sich sogar einzelne Vertreter der Pettenkofer'schen Ansicht durch die letzte Choleraepidemie in Hamburg irre machen lassen, weil Koch und seine Schule behaupteten, in Hamburg sei das Wasser die Ursache der Epidemie gewesen, namentlich deshalb, weil die Cholera an der Grenze von Altona Halt gemacht habe, so daß in einzelnen Straßen z. B. der Straße „Schulterblatt" die zu Hamburg gehörige Straßenseite von der Epidemie schwer heimgesucht wurde, während die zu Altona gehörige gegenüberliegende Häuserreihe fast frei geblieben ist. Allein, meine Herrn, daß zwischen der Hamburger und Altonaer Seite ein ganz gewaltiger Unterschied in bezug auf die Wohnungsverhältnisse besteht, das muf selbst ein Blinder erkennen.

Auf der Hamburger Seite haben alle Häuser Kellerwohnungen und namentlich liegen auch viele Nahrungsmittelverkaufsstellen im Keller. Die Hamburger sind Erdhöhlenbewohner, die Altonaer nicht; denn auf der Altonaer Seite sieht man keine einzige Kellerwohnung.

Außerdem sind die Bodenverhältnisse in Altona und Hamburg grundverscbieden. Hamburg hat in den meisten Stadtteilen, namentlich in St. Pauli, Nord und Süd, Hammerbroock etc. einen für Cholera disponierten Boden, während Altona größtenteils auf Lehmboden liegt, der nach Pettenk ofer bekanntlich choleraimmun ist.

Wer sich davon überzeugen will, daß die Cholera in Hamburg nicht durch das Wasser, sondern durch die Bodenverhältnisse verursacht wurde, der lese Dr. Friedrich Wolter's Schrift: Das Auftreten der Cholera in Hamburg in dem Zeit- 
raume von 1831-1893 mit besonderer Berücksichtigung der Epidemie des Jahres 1892. München, 1898 Verlag von J. F. Lehmann,

Diese Arbeit von Wolter ist um so böher anzuschlagen, als er beauftragt war den Nachweis zu führen, daß die Choleraepidemie in Hamburg durch das Trinkwasser erzeugt wurde. Aber Wolter kam durch gründliche, völlig objektive Untersuchungen zu einem entgegengesetzten Ergebnisse und auf den Pettenk ofer'schen Standpunkt, obgleich er kein Schüler Pettenkofer's ist und mit diesem damals weder persönlich noch schriftlich in Beziehung getreten war. Es gibt Gott sei Dank noch charaktervolle Männer, denen die Wahrheit über alles geht und mit ihrer Hilfe wird es wohl gelingen, der Wahrheit zum Siege zu verhelfen.

Ich glaube heute auch einen bakteriologischen Beweis dafür erbringen zu können, daß die Entstehung von Typhus- und Choleraepidemien durch Brunnen- oder FlußBwasser nicht möglich ist.

Im Laufe der letzten, 20 Jahre wurden gelegentlich des Vorkommens von Typhusepidemien hunderte von Wasserproben aus Brunnen, welche Typhusepidemien verursacht haben sollten, im hygienischen Institut in München bakteriologisch untersucht, aber nicht ein einziges Mal konnten Typhusbacillen in dem verdächtigten Wasser nachgewiesen werden. Einige Bakteriologen wollen zwar neverdings in einigen Fällen Typhusbacillen in derartigem Wasser gefunden haben; allein wir dürfen hinter alle diese angeblich positiven Befunde ein großes Fragezeichen machen, weil die Identifizierung der Typhusbacillen im Wasser auBerordentlich schwierig ist und weil selbst der angebliche Nachweis durch Agglutination noch berechtigte Einwände und Zweifel zuläßt,

Es ist aber auch aus folgenden Gründen höchst unwahrscheinlich, daß Typhusund Cholerabacillen în einem Brunnen länger als 48 Stunden nachweisbar sind, auch wenn ein ganzer Typhusstuhl in emen Brunnen gelangt, was im zivilisierten Deutschland doch kaum denkbar ist.

Bringt man nämlich größere Mengen von Typhusbacillen in Fluß-, Leitungsoder Brunnenwasser, so werden sie rasch in großer Zahl vernichtet.

1 cem Ruhrwasser enthielt nach dem Zusatz von Typhusbacillen :

\begin{tabular}{|c|c|c|c|c|c|}
\hline Sofort & nach dem $Z u$ & atz & . & 21600000 & Typhusbacillen \\
\hline Nach & 44 Stunden & . & . & 7200000 & $"$ \\
\hline$"$ & 66 & . & . & 128571 & $"$ \\
\hline " & 105 & . & . & 0 & $\eta$ \\
\hline
\end{tabular}

$1 \mathrm{ccm}$ Mangfallwasser enthielt am 26. Dezember 1903:

$\begin{array}{cccrc}\text { Sofort nach Zusatz } & . & . & 10543000 & \text { Typhusbacillen } \\ \text { Nach } 24 \text { Stunden } & . & . & 1800000 & " \\ \text { " } 48 \quad, \quad . & . & 0 & "\end{array}$

1 ccm Wasser aus dem Brunnen des Hygienischen Institutes, welches sehr reich an Protozoen, namentlich auch an Flagellaten, sowie an Infusorien, Cyklopiden etc. ist, enthielt am 2. Mai 1904:

Sofort nach Zusatz . . . . . .

Nach 24-stündigem Stehen bei $21^{0}$.

24300000 Typhusbacillen

2885714

Ganz anders verhalten sich die Typhusbacillen in diesen Wasserproben, wenn diese vor Zusatz der ersteren durch 1-stündiges Erhitzen in strömenden Dampf sterilisiert werden. Im sterilisierten Fluß-, Leitungs- und Brunnenwasser findet innerhalb 
5 Tagen weder eine $\mathrm{Zu}$ - noch Abnahme der eingesäten Typhusbacillen statt, oder die letztere ist sehr gering.

$1 \mathrm{ccm}$ sterilisiertes Ruhrwasser enthielt nach dem Zusatz von Typhusbacillen :

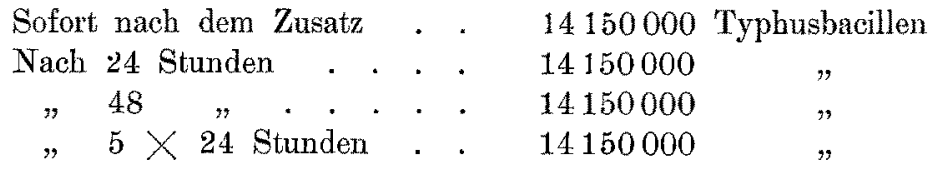

$1 \mathrm{ccm}$ sterilisiertes Mangfallwasser enthielt:

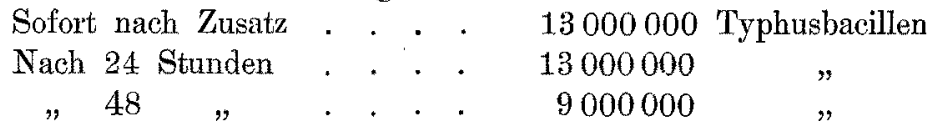

$1 \mathrm{~cm}$ sterilisiertes Brunnenwasser aus Montgelasstrabe 31 enthielt:

Sofort nach dem Zusatz . . 11500000 Typhusbacillen

Nach 24 Stunden . . . 11500000 "

" 48 ". . . . 11000000 "

Im sterilisierten Wasser werden also die Typhusbacillen nicht vernichtet; sie bleiben darin in der ursprünglich eingesäten Menge wenigstens mehrere Tage lebend und entwickelungsfähig, es tritt weder eine Vermehrung noch eine Abnahme der Zahl der Typhusbacillen im sterilisierten Wasser ein.

Durch das Sterilisieren (Erhitzen in strömendem Dampf) wird also irgend etwas im Brunnen- oder Flußwasser zerstört, was vorher vernichtend auf Typhusbacillen wirkte ${ }^{1}$ ).

Sie werden nun fragen, meine Herren, wodurch wird diese enorme Zahl von ins Brumnen- oder Flußwasser eingesäten Typhusbacillen vernichtet? Was ist die Ursache dieses merkwürdigen Vorganges, durch welchen die Natur alle natürlichen Gewässer gegen die Verseuchung durch für Menschen pathogene Bakterien schützt und die Entstehung von Trinkwasserepidemien unmöglich macht?

Die heutige Wissenschaft gibt auf diese Frage eine ganz unrichtige Antwort. Die Bakteriologen behaupten, diese Abtötung der Millionen von Typhusbacillen in jedem ecm Wasser binnen 24 Stunden werde durch die Konkurrenz der Typhusbacillen mit den gewöhnlichen Wasserbakterien, welche stets Sieger bleiben sollen, verursacht.

Dies ist unrichtig; denn wenn man ein reines Wasser z. B. Mangfallwasser, welches nur 4 bis 10 Keime im Kubikcentimeter entbält, solange stehen läßrt, bis es keimfrei geworden ist, dann tötet es gleichwohl Hunderttausende von Typhusbacillen pro $1 \mathrm{ccm}$ in 24 Stunden ab. Also die Wasserbakterien können nicht die Ursache dieses raschen Unterganges der Typhusbacillen sein. Untersucht man aber das von gewöhnlichen Wasserbakterien freie Mangfallwasser mikroskopisch, so findet man, wie ich schon oben erwähnt habe, keinerlei lebende Organismen darin, außer zwei Arten geiBeltragender Flagellaten: Bodo saltans und Bodo ovatus, welche in äuferst lebhafter Bewegung das Wasser durchschwimmen und die Typhusbacillen, wie ich gemeinschaftlich mit Dr. Gemünd festgestellt habe, auffressen und verdauen.

1) Freilich können vereinzelte Typhusbacillen in suspendierte Stoffe eingeschlossen länger im Wasser bleiben; aber an diesen Partikeln haften auch Protozoen, bereit, die frei werdenden Bacillen aufzuzehren. Ich weils aus anderen Beobachtungen, dak ein oder einige Typhusbacillen, in homöopatischer Verdünnung mit Wasser genossen, keine Infektion verursachen und sicherlich können so keine Epidemien entstehen. 
Diese Flagellaten, welche aus dem mit Typhusbacillen versetzten Mangfallwasser gewonnen wurden ${ }^{1}$ ), waren vollgefressen mit Typhusbacillen, jede Flagellate enthielt mehrere Typhusbacillen, oft sogar 5, 6, 8, 10, 20 und noch mehr und man konnte auch deutlich den Zerfall der Typhusbacillen d. h. ihre Auflösung im Flagellatenleib beobachten. Man sab neben mehr oder weniger gut erhaltenen Typhusbacillen nur noch Kernreste und Detritusmassen als Uberbleibsel derselben. Die Typhusbacillen werden also im Flagellatenleib aufgelöst d. h. verdaut. Vergl. Fig. 1 und 2 der Tafel.

Die Natur hat alle Gewässer durch die Flagellaten, die zu den einfachsten, aber schönsten und bewunderungswürdigsten Organismen gehören, gegen die Möglichkeit der Verseuchung durch pathogene Bakterien geschützt und dieser Schutz ist unendlich viel wirksamer und sicherer, als der, welcher durch die Kontrolle der Wasserwerke von seiten der amtlichen Ärzte Deutschlands ausgeübt wird.

Prof. Metsehnik off in Paris hat schon vor mehr als 30 Jahren nachgewiesen, daß auch das Blut der Menscheni und Tiere, sowie deren gesamter Organismus durch einen ganz ähnlichen Vorgang gegen die Infektion durch pathogene Bakterien geschützt werden. Metschnikoff hat gezeigt, daß die weißen Blutkörperchen (Phagocyten) die ins Blut eingedrungenen pathogenen Bakterien auffressen und verdauen, genau in derselben Weise wie die Phagocyten des Wassers, d. h. die Flagellaten die ins Wasser gelangenden pathogenen Bakterien aufnehmen und durch Verdauen vernichten.

Wenn Sie die Abbildungen mit den von Metschnik off gefertigten Bildern, welche die mit pathogenen Bakterien rollgefressenen Phagocyten (weife Blutkörperchen) des Blutes darstellen, vergleichen, so ist die Ähnlichkeit der Formen und die Analogie des Vorganges auch für den Laien in die Augen springend.

Ich höre aber schon jetzt, wie uns die Trinkwasser-Theoretiker zurufen werden: Gerade diese Analogie ist der beste Beweis dafür, daß durch diese Einrichtung der Natur die Entstehung von Trinkwasserepidemien nicht verhütet werden kann, insofern öfter's doch viele pathogene Bakterien ihren Verfolgern, den Flagellaten etc. entgehen und dann zu Infektionen führen können, geradeso wie es trotz der großen Zahl der Phagocyten des Blutes sehr häufig doch zu einer Überschwemmung des Blutkreislaufes mit pathogenen Bakterien und dadurch zur tödlichen Erkrankung kommt!

Nun, meine Herren, dieser Vergleich hinkt ganz gewaltig und dieser Einwand ist leicht zu widerlegen!

Der Hauptgrund für die Haltlosigkeit dieses Einwandes ist die Tatsache, daP sich die pathogenen Bakterien im menschlichen und tierischen Organismus und auch im Blute rapid zu vermehren vermögen, derart, daB in wenig Stunden aus einem Keim Millionen entstehen, so daß die Zahl der Blat-Phagocyten zu ihrer Vernichtung micht mehr ausreicht.

Im Wasser aber ist gerade das Umgekehrte der Fall: im Wasser vermehren sich fortwährend die Flagellaten und Infusorien etc., während sich die pathogenen Bakterien darin nicht zu vermehren vermögen.

Wenn man berechnet, wie viel Typhusbacillen durch die in der gesamten Wassermenge eines Brunnens enthaltenen Flagellaten vernichtet werden, so stellt sich heraus, daß diese Zahl eine ganz unerwartet große, ja geradezu eine enorme ist.

1) Um die Flagellaten in grofer Zahl zu erhalten, setzt man Typhus-Bacillen - eine kleine Öse einer Agar-Kultur auf $100 \mathrm{ccm}$ Wasser - zum Wasser. Nach einigen Tagen centrifugiert man und sucht durch fraktionierte Aussaat in sterilisiertes, aber mit TyphusBacillen versetztes Wasser Reinkulturen der verschiedenen Arten zu erhalten. 
Es wurde schon erwähnt, daß $1 \mathrm{ccm}$ des Wassers vom Brunnen des hygienischen Institutes in München innerhalb 24 Stunden 21414268 Typhusbacillen abtötet.

Die Flagellaten in 1 Liter dieses Wassers vernichten also in 24 Stunden 21414286000 Typhusbacillen.

Es wird selten ein Brunnen zu finden sein, welcher weniger als 100 Liter Wasser enthält. Rechnen wir nun mit diesem Minimum von nur 100 Litern, so ergibt sich, daß die gesante Wassermasse dieses Brunnens die enorme Zahl von 2141428600000 Typhusbacillen innerhalb 24 Stunden vernichten Kann.

Nimmt man an, daß $1 \mathrm{ccm}$ Typhusstuhl 10000000 Typhusbacillen enthält - eine sehr hohe Zahl, die tatsächlich nur selten darin enthalten ist - daun enthält ein ganzer Typhusstuhl von $200 \mathrm{~cm} 2000000000$ und 1000 Typhusstühle enthalten 2000000000000 Typhusbacillen, also weniger als die Wassermenge des Brunnens in 24 Stunden zu vernichten vermag.

Man könnte also - man höre und staune! - tausend Typhusstühle täglich in den Brumnen des bygienischen Institutes werfen, - die darin enthaltenen Typhusbacillen wären bis zum nächsten Tag daraus verschwunden.

Wahrhaftig, gegenüber solchen Zahlen kann nur noch ein blindgläubiger Fanatiker an die Möglichkeit glauben, daß durch Brunnenwasser Typhusepidemien verursacht werden können. Wer aber rechnet und denkt, muß, auch wenn er strenggläubiger Trinkwasser-Theoretiker ist, zugestehen, daß angesichts dieser Tatsachen die Entstehung von Typhusepidemien durch Brunnenwasser eine Unmöglichkeit ist.

Je unreiner im allgemeinen ein Brunnen ist, $d . h$. je mehr Protozoen er enthält, um so mehr Typhusbacillen werden in ihm vernichtet ${ }^{1}$ ).

Geringer ist die abtötende Wirkung eines ganz reinen Wassers; aber selbst das jungfräulichem Boden entstammende Mangfall-Leitungswasser tötet binnen 24 Stunden mindestens 20000 Typhusbacillen pro $1 \mathrm{ccm}$, so daß ein damit gefüllter Brunnen von 100 Litern immerhin die Typhusbacillen eines Typhusstuhles im Tage vernichten würde. Es gibt wenig Brunnen, die eine noch geringere Wirkung haben, so daß wir mit aller Entschiedenheit behaupten können, daß die Entstehung von Typhusepidemien durch Trinkwasser eine Unmöglichkeit ist.

Die Flagellaten finden sich in jedem natürlichen Wasser, selbst in Berg- und Waldquellen habe ich sie gefunden, kaum 300 Meter vom Ursprung entfernt.

Die Quelle, die gerade aus jungfräulicher Erde geboren durch den Wald rauscht, der Gebirgsbach, welcher die Gletscherregion verlassend tosend über die Felsen stürzt, jeder Brunnen, jeder Bach, jeder Fluß und jeder See enthält in großer Zahl diese hygienischen Schutztruppen des Wassers, die Flagellaten, die es gegen jede Verseuchung schützen.

Ihre Keime müssen in der Erde schlummern, bis sie, von den Fluten dahingetragen, zu Millionen kleinster aber auberordentlich bedeutungsvoller Organismen sich entwickeln und vermehren.

Wie wir voll andachtsvoller Bewunderung vor den groBen Zwecken der Milliarden und aber Milliarden von Sternen, Sonnen und Welten stehen, welche den unendlichen Raum mit blitzartiger Geschwindigkeit durcheilen, so beginnen wir staunend die großartige, auch für das Wohl der Menschen so bedeutungsvolle Bestimmung dieser unzähligen kleinsten Wesen der Protozoen, Infusorien etc. zu erkennen.

1) Die meisten Brunnen in kleinen Städten und Ortschaften sind aukerordentlich reich an Protozoen, so daß sie eine ähnlich energische Wirkung auf Typhusbacillen ausüben werden, wie das Wasser des Brunnens im Hygienischen Institut zu München. 
Es freut mich, daß ich über diese Untersuchungen zuerst in Stuttgart berichten durfte, das von jeher durch seine Cholera-Immunität eine Hochburg der lokalistischen Lehre war. Durch neue Untersuchungen über die Ursache der Cholera-Immunität Stuttgarts, die ich mit Dr. Gemünd ausgeführt habe, wird diese Hochburg der Bodentheorie zu einer uneimnehmbaren Feste werden, auf deren Zinnen die Fahne der Wahrheil weht mit der Inschrift: „Nieder.mit der Trinkwasser-Theorie“. - Ja es wäre ein großer Segen, es würde das Glück von Millionen bedeuten, wenn es gelingen würde, die Unhaltbarkeit der Trinkwasser-Theorie darzutun. Denken Sie nur an unsere heldenhaften Brüder in Westafrika, die, mit einem verschlagenen Feinde kämpfend, von der nicht minder tückischen Seuche, dem Typhus, dezimiert werden. In dem falschen Glauben, daß das Trinkwasser die Ursache sei, versäumt man auch dort die allein wirksamen Maßregeln zur Durchführung zu bringen, welche die lokalistische Lehre verlangt - und Unheil über Unheil ist die Folge!

Diese von mir erörterte Frage hat gerade für Sie, meine Herren, d. h. für die Chemiker und speziell die Nahrungsmittelchemiker ein ganz hervorragendes Interesse. Wenn es nämlich wahr ist, daf Typhus- und Choleraepidemien nicht durch Trinkwasser verursacht werden können, dann haben die Ärzte und Hygieniker in Zukunft nicht mehr das Recht, die bakteriologische Untersuchung des Wassers zu monopolisieren und ihre Ausführung sowie die Beurteilung für sich allein zu beanspruchen. Das Wasser ist alsdann in Zukunft lediglich als ein Nahrungsstoff des Menschen bezw. als menschliches Getränk auf seine Reinheit zu untersuchen und die lokalen, chemischen, bakteriologischen und mikroskopischen Untersuchungen, die hierzu nötig sind, können von eimem Chemiker, welcher Bakteriologie und die Flora und Fauna des Süßwassers studiert hat, ebensogut ausgeführt werden wie von einem Arzt und Hygieniker. Nur in den seltenen Fälen, in denen wirkliche Gifte, wie Blei, Sublimat u. dgl. im Wasser nachgewiesen werden, wird man den Hygieniker zu Rate ziehen, abgesehen davon, daß bei solchen Vorkommnissen die Frage der Gesundheitsschädlichkeit eine sehr klare und kaum zweifelhafte sein wird.

\section{Erklärung der Abbildungen.}

Fig. 1. Flagellaten, Bodo saltans (länglich) und Bodo ovatus (rund), aus Mangfall-Leitungswasser durch Zusatz von Typhus- und Prodigiosus-Bacillen gezüchtet. - Vergrößserung 250-fach; Zei\&' Objektiv, Brennweite $8 \mathrm{~mm}$, Okular 8 , Tubuslänge $19 \mathrm{~cm}$.

Fig. 2. Trockenpräparat von Flagellaten, $1 / 2$ Stunde nach Zusatz der Typhusund Prodigiosus-Bacillen zum Wasser. Die Bacillen sind in großer Zahl im Flagellaten-Leibe sichtbar, desgl. auch Zerfallsprodukte derselben. - Färbung nach Gi ems a (Centrbl. Bakteriol. I. Abt. 1902, 32, 307); nur eine Flagellate ist zur Sichtbarmachung der Geißeln mit Eosin gefärbt. - Vergrößerung etwa 700 - fach; Zeifí Homogene Immersion, Brennweite $3 \mathrm{~mm}$, Okular 8 , Tubuslänge $16 \mathrm{~cm}$.

\section{Diskussion.}

Der Vorsitzende dankt den Referenten für ihre interessanten Mitteilungen und fragt Dr. Emmerich, ob er mit den von König aufgestellten Leitsätzen einverstanden sei, namentlich auch mit Leitsatz 1.

Dr. Emmerich erklärt sein Einverständnis und bittet die Stelle seiner Ausführungen „Zur Beurteilung eines Wassers, das für die Versorgung einer Stadt oder Ortschaft, oder eines Komplexes von solchen bestimmt ist, sind die notwendigen hydrotechnischen, geologischen und physikalischen Untersuchungen, die Augenscheinnahme inbegriffen, sowie 
die chemische, bakteriologische und mikroskopische Analyse auszu-

fuhren und so oftzu wiederholen, als dies zur Feststellung dermog.

lichen $8 \mathrm{chwankungen}$ in bezugaf Qualitat und Quantitat notig ist." als weiteren Leitsatz anzufügen. Er bittet ferner der mikroskopischen Untersuchung des Wassers, die in letzter Zeit etwas vernachlässigt werde, wieder mehr Aufmerksamkeit zu schenken.

Dr. Bömer glaubt, daß̧ sich der von Prof. Emmerich vorgeschlagene Leitsatz am besten an den König'schen Leitsatz 6 anreihe.

Dr. Heckmann fragt, ob die Flagellaten nor pathogene, oder auch andere Keime vernichten; andere Keime verschwanden doch nicht aus dem Wasser.

Dr. Emmerich erklärt, dafs auch andere Keime verschwänden, allerdings nirht in dem Maße, wie die pathogenen. Es scheint, daf die Nagellaten in der Tat eine Vorliebe für pathogene Keime besitzen; vielleicht hänge dies auch von der chemischen Beschaffenheit des Wassers ab. Die Flagellaten werden am Brunnengrund fortwährend ergänzt ${ }^{1}$.

Dr. Heckmann schlägt vor, die Vorträge von Geh. Rat König and Prof. Emmerich vervielfältigen und allen Behörden, insbesondere sämtlichen Kreisärzten überreichen zu lassen.

Dr. Medicus bittet dies dem Ausschuß zu überlassen.

Dr. B 0 m er glaubt, daß sich vielleicht auch der Buchhandel der Verbreitung dieser Vorträge annehmen werde.

Bezüglich der Typhusepidemie in Gelsenkirchen habe man deren Crsache in einem Rohrbruch der Wasserleitung gesucht, in dessen Näho ein Typhusfall gewesen sei.

Dr. Emmorich bemerkt dazu, das man in Gelsenkirchen zwei Theorien bezilglich der Typhusepidemie aufgestellt habe; bei den auserordentlich mangelhaften hygienischen Verhälnissen Gelsenkirchens sei es aber nicht $z$ weifelhaft, wo die Ursache des Typhus zu suehen ist. Das Wasserwerk in Gelsenkirchen sei von allen hygienischen Einrichtungen dort noch die beste.

Medizinalrat Dr. S che ur l en erörtert die Wasserversorgungs verhältnisse Württembergs, das auf diesem Gebiete allen deutschen Bundesstaaten vorangegangen sei; der Behauptung von $\mathrm{K} \ddot{\mathrm{n}} \mathrm{ig}$, daß bis vor 15 Jahren die chemische Untersuchung die allein maß3gebende gewesèn sei, müsse er widersprechen. In Württemberg sei die Frage durch eine Zentralwasserversorgung genau geregelt; es wurden an die Gemeinden Formulare hinausgegeben, die von diesen genau auszufullen sind. Man könne in Württemberg nicht verstehen, wie ein solcher Zwiespalt zwischen den chemischen und hygienischen Gutachtern entstehen lonnte, in Wüttemberg seien Differenzen zwischen beiden unbekannt. Er könne nicht zugeben, dak die chemische Wasseruntersuchung ausschlaggebend sei; sehr wichtig sei die berucksichtigung der geologischen Verhältnisse und aus dex geologischen Karte liefen sich schon bestimmte Schlüsse auf die Beschaffenheit eines Wassers ziehen. Auf jeden Fall gehöre zur Beurteilung des Wassers große praktische Erfahrung, die sich der Chemiker wie der Arzt erwerben könne. Die physikalische Untersuchung habe ebensoviel Wert wie die chemische und bakteriologische; aucl qualitative chemische Reaktionen seien nicht ohne Wert nnd es gebe Fälle, wo sie allein genügten. Die bakteriologische Untersuchung sei nur in seltenen Fällen nötig; sie sage oft zar nichts. Er vermöge nicht zuzugehen, daß die chemische Wasseruntersuchung von den Hygienikern geschmaht worden sei, hochstens von ganz unerfahrenen.

Gegentiber den Ausfuhrungen von Prof. Emm erich bemerkt er, dab es nie moglich sein werde, nachzuweisen, daf Typhus und Cholera mit dem Trinkwasser nichts zu tun hätten; in Würtemberg soi die Verbreitung von Typhus durch Wasser wirklich vorgekommen. Die Annahme, je anreiner ein Wasser sei, um so mehr Bacillen zerstöre es, sei nicht verständlich.

Dr. Bömer erklärt, dafi, wenn die Verhältnisse allgemein so lägen, wie sie von dem Herrn Vorredner für Württemberg geschildert seien, Herr Geheimrat König sich jedenfalls vollkommen mit diesen einverstanden erklären würde; in Preaßen lägen aber die Verhältnisse in dieser Beziehung ganz anders.

Dr. E $\mathrm{mm}$ erich glaubt, daß3 sich Herr Medizinalrat $\mathrm{Sch}$ eurlen im allgemeinen ja im Sinne der beiden Referenten ausgesprochen habe. Die Frage der Typhusübertragung könne hier nicht entsehieden werden, tatsächlich sei aber in vielen Fällen, wo anfangs das Wasser als Typhusverbreiter angesehen wurde, diese Ansicht spater widerlegt worden. In unreinem Wasser gehen die Protozoen nicht zu grunde.

Der Vorsitzende bitet uber die Annahme der Leitsatze abzustimmen; es erfolgt einstimmige Annahme.

1) Die wesentliche Ursache, wefhalb im Brunnenwasser die pathogenen Bakterien so rasch von den Protozöen vernichtet werden, die Wasserbakterien aber nicht, liegt wohl darin, daf sich die letzteren im Wasser zu vermehren vermögen, die ersteren aber nicht. 


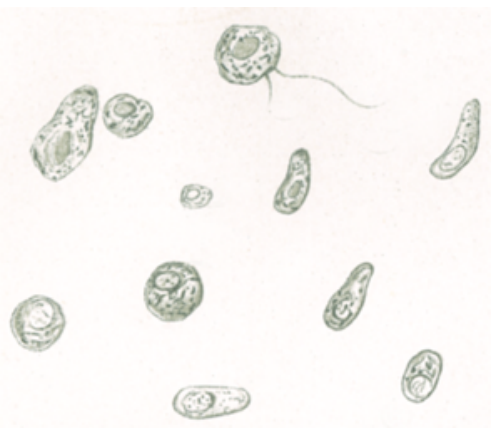

Fig.1.
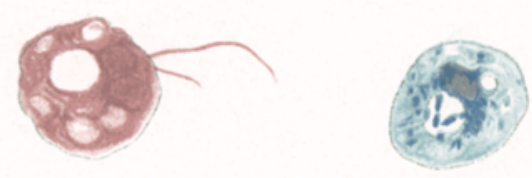

Fig.

Verlag von Jrthus Springer in Berlin. 